Psychological Medicine 2000, 30, 743-746. Printed in the United Kingdom

(C) 2000 Cambridge University Press

EDITORIAL

\title{
The need for treatment evidence for common mental disorders in developing countries ${ }^{1}$
}

There is now a considerable body of epidemiological evidence from developing countries and multilateral agencies that so called common mental disorders are common and disabling (e.g. Murray \& Lopez, 1996; Patel et al. 1998). The logical next step is to identify efficacious and costeffective health services interventions that can tackle the all-important 'so-what' question that is posed by doctors and policy makers in developing countries when confronted with startling prevalence statistics. The rise of evidence-based medicine has highlighted the need for quality trials that can inform and guide clinical practice. This editorial poses the question of whether we need treatment evidence from developing countries. The key issue is whether we can assume that psychiatric treatment evidence can be applied from one culture, or region, to another. If, for example, this was possible, then clinical practice in developing countries could be fairly well informed by trials conducted in the West. However, there are several factors that limit the crosscultural applicability of treatment research in psychiatry.

\section{WHY TREATMENT RESEARCH MAY NOT TRAVEL WELL}

Primary care research on common mental disorders, in particular, may not travel well from one world region to another for a number of reasons.

\section{Cultural factors}

There is now considerable awareness about depression in many Western countries, and many health practitioners and patients recognize it as a mental illness with specific treatment guidelines (Paykel \& Priest, 1992; Paykel et al. 1998). Common mental disorders, however, not only vary considerably in prevalence across different countries, but also in terms of risk factors and the way these disorders are conceptualized by patients and health-care providers (their explanatory models). In many countries, both developing and developed, they are often conceptualized as being related to stress, social and economic problems and not seen from the perspective of biomedical 'mental' disorders. The key difference between developed and developing countries is the attitude concerning the kind of help that is needed to resolve the disorder. Thus, in developed countries, there is a far greater recognition that the disorders are disturbances in health and could benefit some form of medical or psychological intervention. The explanatory models of common mental disorders in many developing countries, on the other hand, are far less likely to acknowledge a role for biomedical interventions (Patel, 1996a; Patel et al. 1998). Such models are likely to influence the acceptability of psychiatric interventions. The cultural influences are likely to be even greater for 'talking' treatments. Clinical evidence suggests that conventional Euro-American models of psychotherapy with their orientation towards individuality and intrapsychic introspection are of limited efficacy with neurotic problems in African and Asian patients (Sethi \& Trivedi, 1982; Swartz, 1987).

1 Address for correspondence: Dr Vikram Patel, The Sangath Centre, 841/1 Alto Porvorim, Goa 403521, India. 


\section{Health systems factors}

Much of the treatment research in the West is based on relatively stable health systems that are underwritten by public or insurance funding. For most patients, health care is free at the point of delivery. By contrast, general health care in many developing countries is often provided by the private sector. In India, for example, it is estimated that more than half of health care is 'out of pocket'. Private practitioners often work alone and outside any organized health care system. In addition, traditional healers play an important role in some countries, particularly in Africa. Mental health manpower in developed countries, though often described as inadequate, is in fact very generous when compared with the situation in developing countries. The few psychiatrists who are available in developing countries are either based in hospitals caring for the severely mentally ill, or in private practice in urban areas catering to the upper classes. The role of non-medical mental health professionals such as nurses and social workers has been well-established in the West; thus studies have shown that cognitive and behavioural therapies can be effectively provided by such professionals. However, if anything, the availability of such professionals in developing countries is even more limited. Non-governmental organizations (NGOs) are becoming important players in mental health care. These are often action-orientated, community-based organizations that are more clearly focused to linking research with service development. Their role in other areas of public health, such as sexual health, has been of great significance and much research and programme development is now being conducted in collaboration with NGOs in developing countries. Social care and assistance, which is a core aspect of the welfare-orientated health systems of developed nations is poorly provided in most developing countries. Furthermore, the health systems of many developing countries are witnessing profound and rapid changes at a pace that has no parallel in contemporary Western society. The rapid reduction in public health expenditure is leading to a massive growth in the private medical sector, rising health costs, and, as many countries introduce health insurance, new and untried models of health care financing. As might be expected these widely varying and changing health-system related factors can profoundly influence the applicability of treatment research (Patel, 2000).

\section{Pharmacokinetic and pharmacodynamic factors}

While it may be assumed that cultural factors are unlikely to influence treatment response for physical treatments such as antidepressants, there is evidence suggesting that the effective dose of antidepressants is lower among non-Caucasians (Marsella \& Westermeyer, 1993); an open trial in Kenya showed that $75 \mathrm{mg}$ of clomipramine daily was as effective as $150 \mathrm{mg}$ (Kilonzo et al. 1994). The acceptability and tolerance of side-effects arising from antidepressants may also vary. For example, a pilot randomized controlled trial examining tolerance to imipramine $150 \mathrm{mg}$, imipramine $75 \mathrm{mg}$ and fluoxetine $20 \mathrm{mg}$ in primary-care attenders in Goa found that $>85 \%$ of subjects in the imipramine $150 \mathrm{mg}$ group did not complete 6 weeks of treatment. Even though the drop-out rate in the fluoxetine group was lower, about $60 \%$ did not continue the medicine up to 6 weeks. Sideeffects were the commonest reason for stopping the medication (Pereira \& Patel, 1998).

\section{Regional drug policies}

A recent review of trials comparing the cost-effectiveness of SSRIs $v$. tricyclic antidepressants concluded that although SSRIs appeared to be safer and better tolerated, these advantages did not justify their extra costs (Hotopf et al. 1996). However, the cost of drugs is strongly influenced by regional economic factors such as the differing interpretations of drug patent rules, the production of generic drugs and the variable dose strengths of pharmaceutical preparations. Thus, in India there are more than half a dozen preparations of fluoxetine and the 'therapeutic' dose of $20 \mathrm{mg} / \mathrm{day}$ costs about as much as $75 \mathrm{mg}$ of imipramine or amitryptiline a day. Furthermore, since the most easily available formulation of imipramine or amitryptiline is $25 \mathrm{mg}$, the patient is faced with the prospect of taking at least three tablets a day to achieve a minimum therapeutic dose, which is also likely to affect compliance. Thus, fluoxetine is as cheap as older tricyclics, and has fewer side-effects 
and lower drop-out rates indicating that it may be more cost-effective in India than in the West (Patel, 1996b). The rapid changes in world trade arrangements will lead to an inevitable change in costs of drugs in developing countries in the years ahead; these will also play a constantly changing influence on key questions of cost-effectiveness.

\section{Public health priorities}

Health services and intervention research in psychiatry cannot be seen out of context to public health priorities. This is especially important for common mental disorders, which typically present in non-psychiatric settings. The low status of mental health in policy and programmes in many countries means that psychiatric research must attempt to build bridges with existing priorities and find ways in which interventions relate to the work of general health workers. Public health priorities in many developing countries cover issues as diverse as sexually transmitted diseases and AIDS, violence, reproductive health and poverty alleviation. Mental health issues are clearly relevant in all these priorities and treatment evidence is needed from within these contexts. Examples of such intervention orientated themes could include evaluating the efficacy of a psychotherapeutic intervention to improve mental health outcomes in victims of family violence and the efficacy of micro-credit schemes whose objective is to minimize informal debt in rural areas in reducing rates of depression and suicidal behaviour. Such research themes can be integrated into existing health and development agendas at little additional cost and demonstrate the practical value of incorporating a mental health paradigm in public health. Treatment research done from a mainly psychiatric context, on the other hand, often serves to reinforce the notion that these disorders are not general health problems at all, but need specialist psychiatric intervention.

\section{Local health policy factors}

Local policy is mostly influenced by local research. This is well demonstrated by the drug policies in many countries, which require at least Phase IV trials before new medications are licensed for use, even if they are being widely used elsewhere. The low status of mental health in national health policy and for international donor agencies means that neither research nor service development in mental health receives much credit. Furthermore, on a political level, just as research from a developing country is unlikely to influence health policy in a European country, the converse also holds true.

\section{TREATMENT RESEARCH TO MEET MENTAL HEALTH NEEDS IN DEVELOPING COUNTRIES}

Thus, there is a need for generating efficacy and cost-effectiveness evidence from different regions of the world. However, there is still little evidence of this kind available today. Recent meta-analyses of antidepressants, for example, included not a single study from a developing country (Song et al. 1993; Hotopf et al. 1997). The availability of evidence on the use of psychological interventions in primary or general health care settings is even less likely; one of the few randomized controlled trials of psychological treatments in a developing country appears in this issue of Psychological Medicine (Sumathipala et al., see pp. 747-757). Of course, it can be argued that the validity of treatment research across cultures applies equally to physical disorders. However, the relative gap between developed and developing countries in treatment provision is less for these disorders for three key reasons: first, physical illnesses command a more privileged status in health policy; secondly, explanatory models often associate these disorders with biomedical treatments; and thirdly, there is a relatively greater volume of treatment research from developing countries for physical disorders because of greater numbers of research professionals and funds. It is also arguable that the developed world is not homogenous and that treatment research in one developed country cannot be automatically applied to another. However, as the meta-analyses quoted earlier show, trials exist from a wide number of developed countries, most developed countries share considerable health system and cultural factors and the general status of mental health in terms of manpower and policy 
is relatively similar. Thus, treatment research is not needed, neither is it feasible, from every region and culture in the world; rather, evidence can be shared between regions that share similar health systems. A model which categorizes various health systems into 'families' that share many political, economic, health services and culture has been described elsewhere (Patel, 1999).

It has been nearly 10 years since the first reports from multilateral agencies identified mental disorders as major public health problems in developing countries. The much-replicated findings of high prevalence, low recognition and inappropriate treatment with hypnotics and symptomatic medicines have now become a mantra in psychiatric conferences and articles. 'Ignorance' and 'lack of awareness' are often cited by psychiatrists as reasons to describe the prescribing habits of primary health workers or the lack of funding and policy interest in developing countries. However, a major factor, which is impeding the use of more appropriate interventions or a greater prominence to mental illness in policy, is the lack of treatment evidence and the tendency for research to be focused on psychiatrists and psychiatric contexts. Arguably, if there was evidence that treatments were efficacious and cost-effective, and that they were clearly linked to other community health problems, then they would be more widely adopted by health workers and health policy makers. It is clearly time, then, to move on from head-counts and surveys demonstrating more prevalence figures and associations. These surveys have achieved, to some extent, the important goal of putting mental illness on the public health agenda. Future psychiatric research in developing countries needs to be more action-oriented, for example in the form of actual intervention trials or studies with the explicit goal of influencing the integration of mental health care in existing community health services and public health priorities. The research must be sensitive to the local needs and involve active participation from potential users of the findings. In selecting settings for intervention research, a variety of health systems should be considered to ensure that findings can be generalized to many regions of the world. Until we gather a body of evidence that demonstrates that something useful can be done for patients with common mental disorders, it is unlikely that health policy and practice in developing countries will respond to meet the needs of the large numbers of mentally ill persons that epidemiological surveys regularly throw up.

VIKRAM PATEL

The author is currently conducting treatment research for common mental disorders in India supported by a Wellcome Trust Tropical Health Services Project grant.

\section{REFERENCES}

Hotopf, M., Lewis, G. \& Normand, C. (1996). Are SSRIs a costeffective alternative to tricyclics? British Journal of Psychiatry 168, 404-409.

Hotopf, M., Hardy, R. \& Lewis, G. (1997). Discontinuation rates of SSRIs and tricyclic antidepressants: a meta-analysis and investigation of heterogeneity. British Journal of Psychiatry 170, 120-127.

Kilonzo, G., Kaaya, S., Rweikiza, J., Kassam, M. \& Moshi, G. (1994). Determination of appropriate clomipramine dosage among depressed African outpatients in Dar es Salaam, Tanzania. Central African Journal of Medicine 40, 178-182.

Marsella, A. \& Westermeyer, J. (1993). Cultural aspects of treatment: conceptual, methodological, and clinical issues and directions. In Treatment of Mental Disorders: A Review of Effectiveness (ed. N. Sartorius, G. de. Girolamo, G. Andrews, G. A. German and L. Eisenberg), pp. 391-418. American Psychiatric Press: Washington, DC.

Murray, C. \& Lopez, A. (1996). The Global Burden of Disease. Harvard School of Public Health: Boston.

Patel, V. (1996a). Recognizing common mental disorders in primary care in African countries: should 'mental' be dropped? Lancet 347, 742-744.

Patel, V. (1996b). Influences on cost-effectiveness. British Journal of Psychiatry, 169, 381

Patel, V. (2000). Health systems research: a pragmatic model for meeting mental health needs in low-income countries. In The
Unmet Need for Treatment (ed. G. Andrews and S. Henderson), pp. 353-377. Cambridge University Press: Cambridge.

Patel, V., Pereira, J., Fernandes, J. \& Mann, A. (1998). Poverty, psychological disorder and disability in primary care attenders in Goa, India. British Journal of Psychiatry 172, 533-536.

Paykel, E. S. \& Priest, R. (1992). Recognition and management of depression in general practice: consensus statement. British Medical Journal 305, 1198-1202.

Paykel, E. S., Hart, D. \& Priest, R. G. (1998). Changes in public attitudes to depression during the Defeat Depression Campaign. British Journal of Psychiatry 173, 519-522.

Pereira, J. \& Patel, V. (1998). Which antidepressant is best tolerated? A randomized trial of 3 antidepressant treatments for common mental disorders in primary care in Goa. Indian Journal of Psychiatry 41, 358-363.

Sethi, B. \& Trivedi, J. (1982). Psychotherapy for the economically less privileged classes (with special reference to India). Indian Journal of Psychiatry 24, 318-321.

Song, F., Freemantle, N., Sheldon, T., House, A., Watson, P., Long, A. \& Mason, J. (1993). Selective serotonin reuptake inhibitors: meta-analysis of efficacy and acceptability. British Medical Journal 306, 683-687.

Sumathipala, A., Hanwella, R., Hewege, S. \& Mann, A. (2000). Randomized controlled trial of cognitive behaviour therapy for repeated consultations for medically unexplained complaints: a feasibility study in Sri Lanka. Psychological Medicine 30, 747-757.

Swartz, L. (1987). Transcultural psychiatry in South Africa (Part 2). Transcultural Psychiatric Research Review 24, 5-30. 